\title{
The Prevalence of Self-Reported Psychological Characteristics of Adults with Lower Limb Tendinopathy
}

\author{
J. Mest ${ }^{1}$, B. Vaughann ${ }^{2,3}$, J. Mulcahy ${ }^{4}$, P. Malliaras ${ }^{5}$ \\ 1 University of Canberra Health Clinics, Faculty of Health, University of Canberra, Canberra, Australia \\ 2 Department of Medical Education, University of Melbourne, Melbourne, Australia \\ 3 School of Health \& Human Sciences, Southern Cross University, Lismore, Australia \\ ${ }^{4}$ College of Health and Biomedicine, Victoria University, Melbourne, Australia \\ ${ }^{5}$ Department of Physiotherapy, School of Primary and Allied Health Care, Monash University, Melbourne,
} Australia

\section{CORRESPONDING AUTHOR:}

Jack Mest

UC Health Clinics

Faculty of Health

University of Canberra

11 Kirinari Street

Bruce ACT 2617

Canberra, Australia

E-mail: Jack.Mest@canberra.edu.au

DOI:

10.32098/mltj.04.2020.14

LEVEL OF EVIDENCE: 3B

\begin{abstract}
SUMMARY
Purpose. There is an emerging body of literature describing psychological associations with lower limb tendinopathies. The literature suggests that those experiencing a lower limb tendinopathy are likely to experience varying degrees of kinesiophobia, depression and catastrophisation. These studies have typically been confined to one lower limb tendinopathy. The current study sought to explore whether these psychological influences were experienced across a range of lower limb tendinopathies in a clinical practice setting.

Materials and methods. The current study utilised a cross-sectional cohort design to explore associations between those presenting with any lower limb tendinopathy and psychological factors. Consecutive patients attending a private physiotherapy practice in Melbourne (Australia) were invited to participate. Those who chose to participate were invited to complete a health questionnaire along with the Hospital Anxiety \& Depression Scale (HADS), Tampa Scale of Kinesiophobia and the Life Orientation Test - Revised.

Results. Ninety-one patients were recruited, with just over half identifying as male, and more than half experiencing a tendinopathy for more than twelve months. Nearly two-thirds $(63.7 \%)$ of the cohort demonstrated kinesiophobia $(n=58,63.7 \%)$. Patients were classified as depressed in $13 \%$ of cases while $21 \%$ were classified as anxious.

Conclusions. Routine screening for kinesiophobia may be valuable for patients presenting with any lower limb tendinopathy. The results also support the potential value of screening patients for the presence of anxiety and/or depression. The extent to which these psychological influences are associated with individual patient's experience of lower limb tendinopathy, requires further exploration, as does the development of these influences over the duration of the tendinopathy.
\end{abstract}

\section{KEY WORDS}

Kinesiophobia; tendinopathy; psychological; anxiety; depression; optimism.

\section{BACKGROUND}

Tendinopathy is characterised clinically by tendon pain and loss of function $(1,2)$. The aetiology of tendinopathy is multifactorial and imbalance between load demands placed on the tendon and its ability to remodel is considered a major factor (3). Other factors that influence the capaci- ty of the tendon to remodel and increase the risk of developing tendinopathy include older age, genetic profile, and metabolic factors such as elevated cholesterol or diabetes $(3,4)$. Once established, the tissue changes in tendinopathy include matrix degradation, characterised by inferior quality and disorganised collagen, accumulation of hydrophil- 
ic proteoglycan molecules that increase bound water and swelling, as well as ingrowth of blood vessels and nerves (2). Breakdown in the endotendon, including degradation of the interfasicular matrix, has also been shown to limit the fatigue resistance of energy storage tendons, which may explain why aging tendons are more susceptible to injury (5). A major challenge in the management of tendinopathy is an incomplete understanding of pain mechanisms and factors that influence tendon pain (6), including psychological determinants that effect chronicity and recovery.

The role of psychological disorders such as depression, anxiety, catastrophisation and kinesiophobia is well established in chronic musculoskeletal pain states, $(7,8)$ and their influence on, or association with, lower limb tendinopathies is beginning to emerge in the literature. A recent systematic review of cross-sectional and prognostic studies suggests that kinesiophobia, depression, stress and catastrophisation are positively associated with plantar heel pain (9). Kinesiophobia, catastrophisation and depression have also been associated with increased symptom severity in gluteal tendinopathy (10), with kinesiophobia contributing to suboptimal outcomes in Achilles tendinopathy (11). These associations have led to the suggestion that sensitisation of the nervous system, and impaired pain processing, may explain persistent tendinopathy pain states, and ongoing loss of function that can occur following tissue-based intervention in tendinopathy (12).

Although there have been a number of recent studies investigating the role of psychological factors in people with lower limb tendinopathy (9-11), to our knowledge, no current study has assessed its prevalence in a consecutive cohort of patients. The aim of this study was to explore the prevalence of psychological factors in a cohort of consecutive patients seeking physiotherapy care for a lower limb tendinopathy. This work also sought to describe the characteristics of the patient cohort and how these psychological factors correlated with these characteristics.

\section{METHODS}

In this cross-sectional study, data was collected on a cohort of consecutive patients presenting for treatment for lower limb tendinopathy at a single physiotherapy clinic in Melbourne, Australia. All patients were managed by a single physiotherapist (PM) at this centre, who has specialised in tendinopathy management for 15 years. Patients were either self-referred or referred by other health professionals (general practitioners, sports and exercise medicine physicians, orthopaedic surgeons, physiotherapists, osteopaths, chiropractors). Data was collected over 18 months between July 2016 and December 2017. The study was approved by the Victoria University Human Research Ethics Committee (HRE16-079), consistent with the journal recommendations (13). All participants provided informed consent.

\section{Participants}

Participants were either referred to the specialist physiotherapist or elected to attend themselves, for a possible tendon-related complaint. Patients were required to be over the age of 18 years and able to read English at a year 7 (12 years of age) level. The clinic administrative staff recruited participants for the study, the practitioner was not aware if the patient they were treating was a participant or not. Non-participation in the study did not influence the opportunity to receive care.

\section{Questionnaires and measures}

Potential participants were provided with a tablet that contained an online version (Qualtrics, Utah, USA) of the survey. The first part of the survey included the information sheet and consent process. Participants who agreed to participate were asked to complete the questionnaires prior to their consultation with the physiotherapist.

Participants completed a range of demographic and health behaviour questions, in addition to single item screening questions for general health ("Please rate your general health"), and life satisfaction ("Overall, how satisfied are you with your life?”) (14). Participants were also asked to complete questionnaires exploring psychological factors outlined below.

\section{Hospital Anxiety and Depression Scale (HADS)}

The HADS was originally developed by Zigmond and Snaith as a self-report tool to detect and measure the severity of depression and anxiety (15). The HADS-D was originally developed based on the symptoms of anhedonia, whilst the HADS-A was based on the developer's research on anxiety and the Hamilton Anxiety Scale $(15,16)$. It has two separate subscales for each emotional disorder and was originally intended for use in a hospital outpatient setting (15). It is extensively used with psychiatric, medical, rheumatological and chronic pain patients (16). The HADS (15) comprises 14 items rated from 0-3 divided into two subscales: anxiety (7 items) and depression (7 items), scores range from $0-21$ for each subscale. A total score is generated for each of the anxiety and depression subscales. The HADS subscales are analysed separately, scores from 8-10 indicate a possible clinical disorder and scores 11-21 a probable clinical disorder (17). Scores greater than 11 are 
used to identify patients with anxiety or depression. The HADS has been found to be an effective tool in the detection of anxiety and depression $(18,19)$ with a sensitivity and specificity of approximately 0.8 (17) and more than acceptable internal consistency with Cronbach alpha ranges from 0.78-0.93 for the HADS-A and 0.82-0.90 for the HADS-D $(16,17)$. It has been concluded that the HADS has both high clinical and research usability to identify the cognitive symptoms of anxiety and depression, in addition to differentiating between the two disorders $(16,17)$. Johnston, Wright and Weinman (20) have proposed that four score ranges can be used to classify the presence and severity of anxiety or depression: 0-7 normal, 8-10 mild, 11-14 moderate and 15-21 severe.

\section{Tampa Scale for Kinesiophobia (TSK)}

The Tampa Scale for Kinesiophobia (TSK) was originally developed to measure fear of movement and its current use has retained its original scoring format. The TSK is a seventeen-item scale used to subjectively measure fear of movement (21) and unhelpful beliefs about pain. The scale is based on the model of fear avoidance, fear of work-related injury and fear of reinjury (22). The TSK has 17 items rated on a 4-point Likert-type scale. Total score ranges from 17 to 68 with a cut off score of 37 or over being considered a high score (22). Four items on the questionnaire are inversely worded and thus negatively scored. Several studies have shown the TSK to be a valid and reliable psychometric measure $(21,23,24)$ with high internal consistency (Cronbachs alpha=0.84) (25). The TSK has been found to be significantly correlated with other scales that measure pain catastrophization and fear of movement which suggests that it is a valid measure of these constructs (25). The TSK was scored according to published cut-off scores (22).

\section{Life Orientation Test-Revised (LOT-R)}

The Life Orientation Test-Revised (LOT-R) (26) measures dispositional optimism or pessimism. There are ten items: three that measure optimism, three pessimism and four fillers which are ranked by the participant but are not included in the scoring. Agreement or otherwise with a statement is rated on a 5-point Likert-type scale, with a higher score being reflective of greater optimism (27). The LOT-R scoring is a continuous dimension and there is no defined cut-off. The original Life Orientation Test was first published in 1985 as a measure of dispositional optimism, which is characterized as an expectation in people that good things will happen $(28,29)$. The revised version (LOT-R) was later developed to provide a more realistic representation of optimism, taking into account the effect of optimism on other health outcomes (28). In the revised version, two items from the original LOT were removed (28). It is primarily a research instrument rather than a clinical measure of the positive trait. Psychometric properties of the revised scale have been shown to be satisfactory (Cronbachs alpha: optimism 0.70 , pessimism 0.74 , total score 0.68 ) and its use supported to measure pessimism and optimism as independent constructs (28).

\section{Inclusion criteria}

Tendinopathies of five different lower limb tendons were accepted for inclusion in the study: the gluteal tendon, proximal hamstring tendon, patella tendon, Achilles tendon and plantar fascia. Although the plantar fascia transcends the typical definition of a tendon as it does not connect muscle to bone (rather fascia to bone), it does still display characteristics that are consistent with tendinopathy on ultrasound (hypechogenicity and thickening) and in response to loading programs (30). It is for this reason that plantar fasciopathy was considered a tendinopathy in this study. Participants were retained if they had comorbidity or secondary musculoskeletal diagnoses, provided that the lower limb tendinopathy was the primary complaint for which they sought care. Potential participants were only excluded following data collection if clinical examination revealed that their pain was not tendon related or if questionnaires were incomplete.

\section{Tendinopathy diagnosis}

Tendinopathy diagnosis was based on a combination of clinical presentation and tests, as is recommended by expert opinion and consensus (Supplementary file 1) (31-35). A single physiotherapist undertook all diagnoses for each participant. Participants were asked to report the location of pain they experienced during the loading tests undertaken during the diagnostic process. Diagnostic imaging was not a prerequisite of diagnostic classification and this is consistent with recommended practice (36). Differential diagnoses for each tendinopathy site were considered using validated tests where possible (Supplementary file 2). Participants were excluded if an alternative diagnosis (not tendinopathy) was their main pain complaint. Participants were retained in the cohort if they had a comorbid pain state (e.g. sacroiliac joint-related pain), but this was not their primary pain complaint.

\section{Pain descriptors}

Self-reported duration of tendon pain was reported on a 4-point scale: 0-4 months, 5-8 months, 9-12 months and 
greater than 12 months. Patients were asked several questions about their symptom behaviour and pain severity. The percentage of the day that participants experienced pain was rated $(0-25 \%, 26-50 \%, 51-75 \%, 76-100 \%)$. Participants rated the average pain severity over the last 7 days on a visual analogue scale $(0-100,100=$ worst pain imaginable) during rest as well as activities that are commonly associated with pain for each tendinopathy (table I). Scores for these questions were then averaged to provide a total score for pain severity that was used in analyses. These activities and questions were adapted from validated pain and function questionnaires where possible (37-40). We did not use validated pain and function questionnaires as they were not available for every tendinopathy included in the current study.

\section{Statistical analysis}

Data were exported from Qualtrics to SPSS (IBM Corp USA, version 24) for analysis. Each completed patient response was screened and the tendon diagnosis added. Descriptive statistics were generated for each of the demographic and health information variables and reported for each tendinopathy diagnosis. Each of the HADS, LOT-R and Tampa were scored according their respective instructions. Results of each questionnaire were coded to reflect the classifications for the HADS (anxious or depressed), LOT-R (optimism or pessimism) and Tampa (kinesiophobia) questionnaires. Inferential statistics (Spearman's rho) were used to investigate the relationship between psychological questionnaires and pain dimensions (duration, activity pain severity, rest pain severity). Descriptive statistics

Table I. Questions about pain severity.

\begin{tabular}{ll}
\hline Site of tendinopathy & Aggravating activity \\
\hline Gluteal & Arising after prolonged sitting \\
& Walking \\
& Sleep \\
\hline Proximal hamstring & Sitting \\
& Lunging \\
& Start of run/walk \\
& Running/walking faster \\
\hline Patellar & Going downstairs \\
& Sitting \\
& Jumping \\
\hline Achilles & Stiffness in the morning \\
& Start of run/walk \\
\hline Plantar fascia & Stiffness in the morning \\
& Start of run/walk \\
& Prolonged standing \\
\hline
\end{tabular}

(mean, median, standard deviation, percentage) were generated for each questionnaire and the internal structure evaluated using Cronbach's alpha. Alpha was set at $\mathrm{p}<0.05$ and effect sizes were calculated where relevant.

\section{RESULTS}

\section{Demographics and health behaviours}

One hundred and thirty-eight $(\mathrm{n}=138)$ consecutive patients were invited to participate with ninety-one $(n=91)$ agreeing (65.9\% response rate). The median age range of the cohort was $45-49$ and comprised 50 men $(55 \%)$ and 41 women $(45 \%)$. More than half of the participants reported tendon pain for greater than 12 months (figure 1). Eighty-six (95\%) participants spoke English at home, while only $8 \%(n=7)$ lived alone. Eighty $(88 \%)$ participants had private health insurance, 26\% ( $\mathrm{n}=24)$ had a health care card, and 70\% $(n=64)$ had university education or higher. The median amount of sleep per night was between 7 and 8 hours and the median exercises per day was between 30 and 59 minutes. Fifty-seven participants $(63 \%)$ self-assessed their general health as very good or excellent. Table II demonstrates the descriptive and internal consistency statistics for the psychological measures.

Table III shows demographic and pain related data for the tendinopathy presentations. Achilles tendinopathy accounted for nearly half of the patient cohort $(n=43,47.3 \%)$, and over half of the patient cohort had experienced their tendon complaint for greater than 12 months ( $\mathrm{n}=48,52.7 \%$, figure 1). Plantar fasciopathy and gluteal tendinopathy were most prevalent in the 50 and over age group, whilst proximal hamstring, patellar and Achilles tendinopathy were

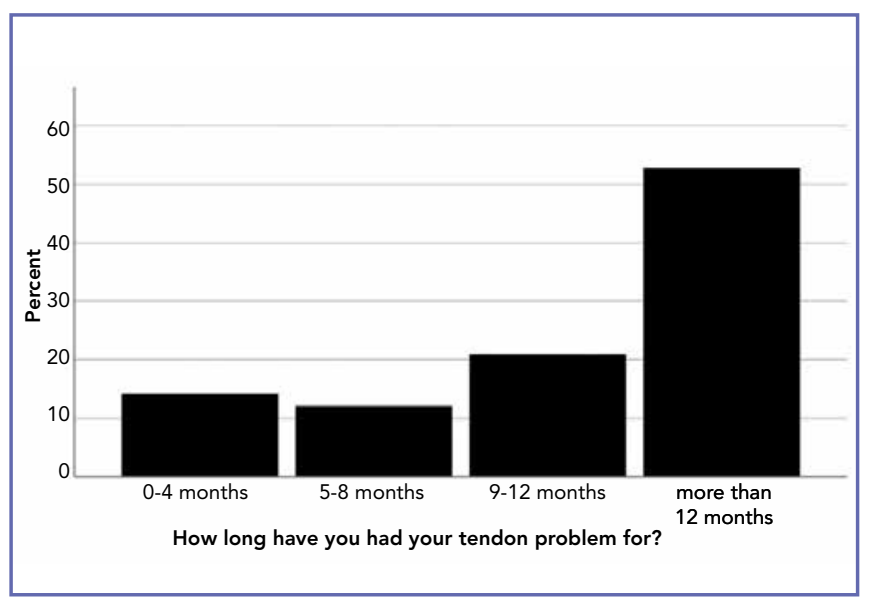

Figure 1. Duration of tendon pain across the entire cohort.

Muscles, Ligaments and Tendons Journal 2020;10 (4) 
Table II. Descriptive statistics and internal consistency for each of the psychological measures.

\begin{tabular}{llll}
\hline Psychological measure & Mean $($ SD) & Range & Cronbach's alpha \\
\hline Tampa Scale for Kinesiophobia (TSK) & & & \\
\hline Somatic Focus & $11.2(3.0)$ & $5-18$ & 0.76 \\
\hline Activity Avoidance & $12.5(3.0)$ & $6-22$ & 0.83 \\
\hline Life Orientation Test -Revised (LOT-R) & & \\
\hline Optimism & $11.5(1.8)$ & $7-15$ & 0.70 \\
\hline Pessimism & $6.6(1.9)$ & $3-12$ & 0.71 \\
\hline Hospital Anxiety and Depression Scale (HADS) & & & \\
\hline Depression & $3.9(2.8)$ & $0-16$ & 0.74 \\
\hline Anxiety & $5.5(2.6)$ & $0-13$ & 0.65
\end{tabular}

Table III. Demographic and symptom data for each tendinopathy.

\begin{tabular}{lllll}
\hline Site of tendinopathy & Cases (\%) & $\begin{array}{l}\text { Most common age } \\
\text { range (yrs) }\end{array}$ & $\begin{array}{l}\text { Percentage of men with } \\
\text { this tendinopathy (\%) }\end{array}$ & $\begin{array}{l}\text { Presence of secondary } \\
\text { diagnosis (\%) }\end{array}$ \\
\hline Gluteal & $11(12.1)$ & 50 or over & 9 & 27 \\
\hline Proximal hamstring & $17(18.7)$ & $35-39$ & 56 & 41 \\
\hline Patella & $13(14.3)$ & $30-34$ & 86 & 46 \\
\hline Achilles & $43(47.3)$ & $35-39$ & 60 & 35 \\
\hline Plantar fascia & $7(7.7)$ & 50 or over & 43 & 29 \\
\hline
\end{tabular}

more common in adults in their thirties (table III). Gluteal tendinopathy was more prevalent in women $(91 \%$ vs $9 \%$, $\mathrm{p}<0.05)$, whilst patellar tendinopathy was more prevalent among men $(86 \%$ vs $14 \%$, p < 0.05$)$.

Patellar tendinopathy was the lower limb tendinopathy most likely to co-exist with a secondary condition (figure 2), while gluteal and plantar fasciopathy were most likely to exist in isolation. Musculoskeletal secondary diagnoses included: hip osteoarthritis, knee osteoarthritis, patellofemoral pain syndrome, sacroiliac joint dysfunction, lower back pain and multiple tendinopathy sites. Systemic concomitant diagnoses included: hypertension, nephropathy, fibromyalgia and psoriatic arthritis. Neurological secondary diagnoses included: sciatic neuropathy and sural neuropathy.

\section{Symptoms}

Participants rated their average pain with activity and with rest (figure 3). In addition, duration of tendinopathy-related pain was reported (figure 4). Achilles tendinopathy was on average the most painful tendon with activity and generated the highest average pain scores at rest. Patellar tendinopathy and hamstring tendinopathies were the least painful tendons at rest. Patellar tendinopathy was also on average

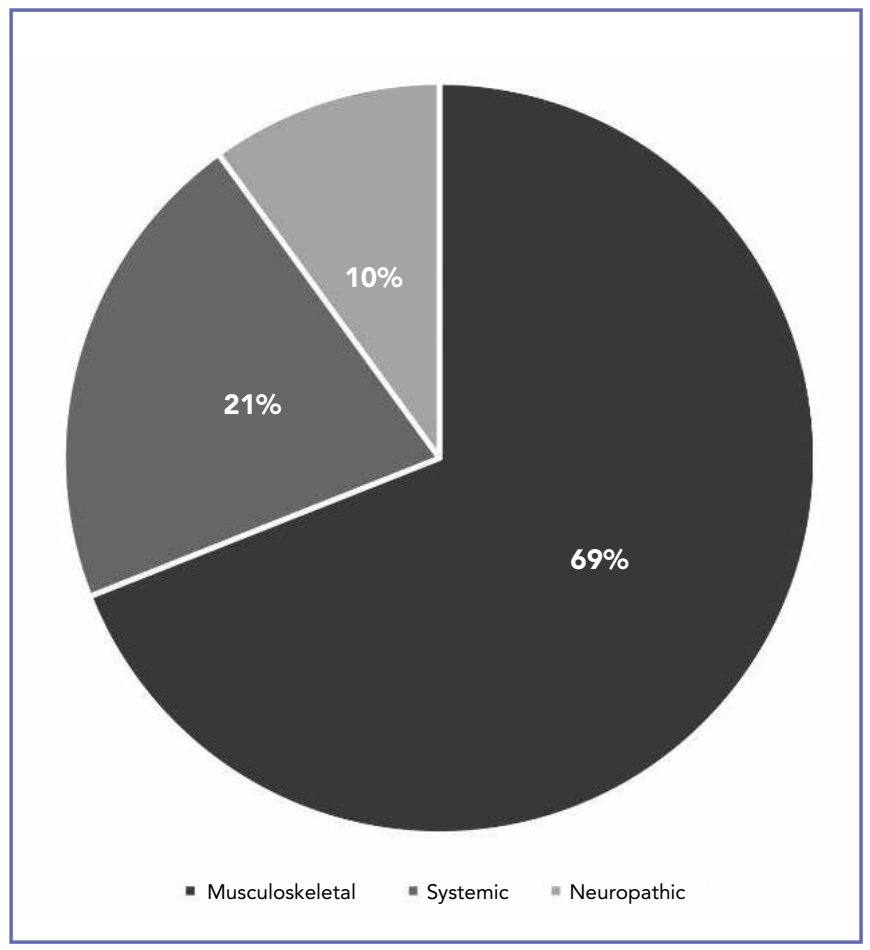

Figure 2. Co-existing diagnoses associated with tendinopathy presentations. 


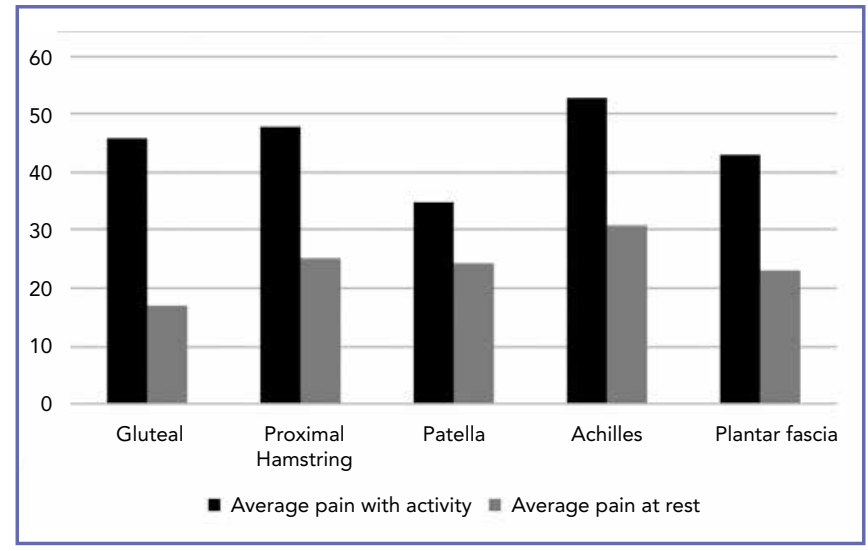

Figure 3. Average pain severity for each tendon subgroup.

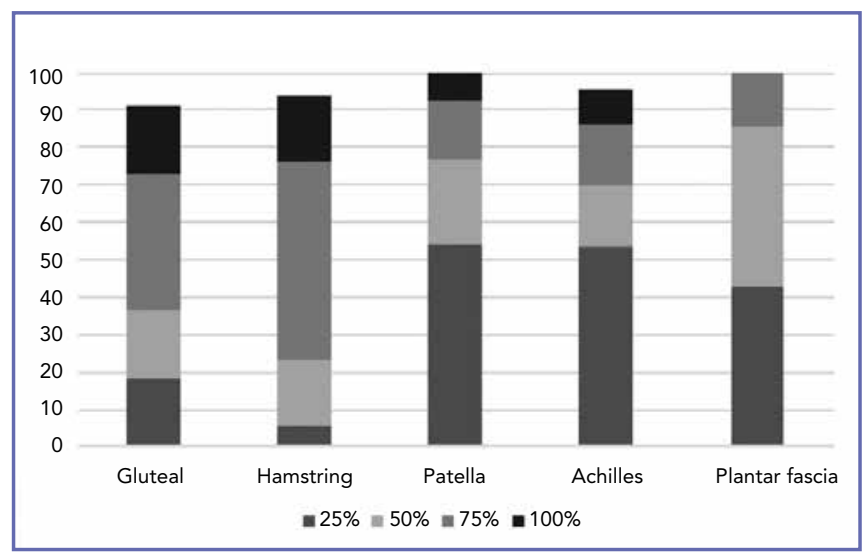

Figure 4. Pain experienced as a percentage of the day for each tendon subgroup.

the least painful condition during activity. Gluteal tendinopathy displayed the greatest discrepancy between pain with activity and rest pain.

The gluteal tendinopathy subgroup demonstrated the highest proportion of participants that experienced pain $100 \%$ of the day $(18.2 \%)$. Over half of the hamstring tendinopathy subgroup experienced pain for three quarters on an average day $(52.2 \%)$. The patellar tendinopathy and Achilles tendinopathy subgroups had the highest proportion of participants that experienced pain for $25 \%$ of the day. Participants with plantar fasciopathy were the only subgroup to not have participants that experienced pain for $100 \%$ of the day.

There was a negative association between self-rated general health and the LOT-R pessimism subscale $(r h o=-0.40, \mathrm{p}<$ 0.05 , medium) and a positive association with the LOT-R optimism subscale $(r h o=0.23, \mathrm{p}<0.05$, small). Small negative correlations were observed between general health and both TSK subscales $(r h o=-0 .-25, \mathrm{p}<0.05$, small), and the HADS depression subscale ( $r h o=-0.23, \mathrm{p}<0.05$, small). Life satisfaction was positively associated with the LOT-R optimism subscale ( $r h o=0.36, \mathrm{p}<0.05$, small). The HADS subscales were negatively associated with satisfaction with life (depression, $r b o=-0.39, \mathrm{p}<0.05$, medium; anxiety, $r h o=$ $-0.25, \mathrm{p}<0.05$, small), however trivial associations were observed between kinesiophobia and life satisfaction.

\section{Tendinopathy and psychological variables}

Nearly two-thirds of the entire cohort scored above the TSK cut-off score $(n=58,63.7 \%)$ with $76.9 \%(n=10)$ of those with patellar tendinopathy classified as having a fear of movement (figure 5). Further, $13 \%$ of participants appear to be affected by depression, while $21 \%$ were classified as anxious. Those patients with gluteal and hamstring tendinopathy exhibited the highest rates of anxiety across the cohort.

Small negative relationships were observed between rest pain severity and the HADS depression subscale $(r h o=-0.22$, $\mathrm{p}<0.01$, small) and TSK activity avoidance subscale ( $r h o=-$ $0.19, \mathrm{p}<0.05$, small) scores. The TSK activity subscale also demonstrated a small relationship to the severity of pain with activity ( $r h o=-0.21, \mathrm{p}<0.05$, small). Symptom duration demonstrated trivial correlations with the subscales on all three psychological questionnaires $(r h o<0.11)$ and LOT-R subscales demonstrated trivial correlations with rest pain and activity pain intensity $(r b o<0.10)$.

\section{DISCUSSION}

This study sought to evaluate the presence of a range of psychological factors that may be associated with the presence of a tendinopathy affecting the lower limb, in a cohort of patients attending for care at a private physiotherapy clinic. Patients in our cohort who were seeking treatment by a physiotherapist with tendinopathy expertise, had undertaken previous treatment (including exercise, injections, and surgery), and 50\% reported they had experienced lower limb tendon pain for longer than 12 months. Further, our cohort was from a high sociodemographic population with $88 \%$ having private health insurance and $70 \%$ having a tertiary education.

\section{Kinesiophobia}

The main finding of our study is that kinesiophobia was highly prevalent across the current cohort regardless of tendinopathy location. Sixty-three percent of all participants displayed beliefs suggestive of fear of movement. This was based on published cut-off scores for the TSK (22) with 


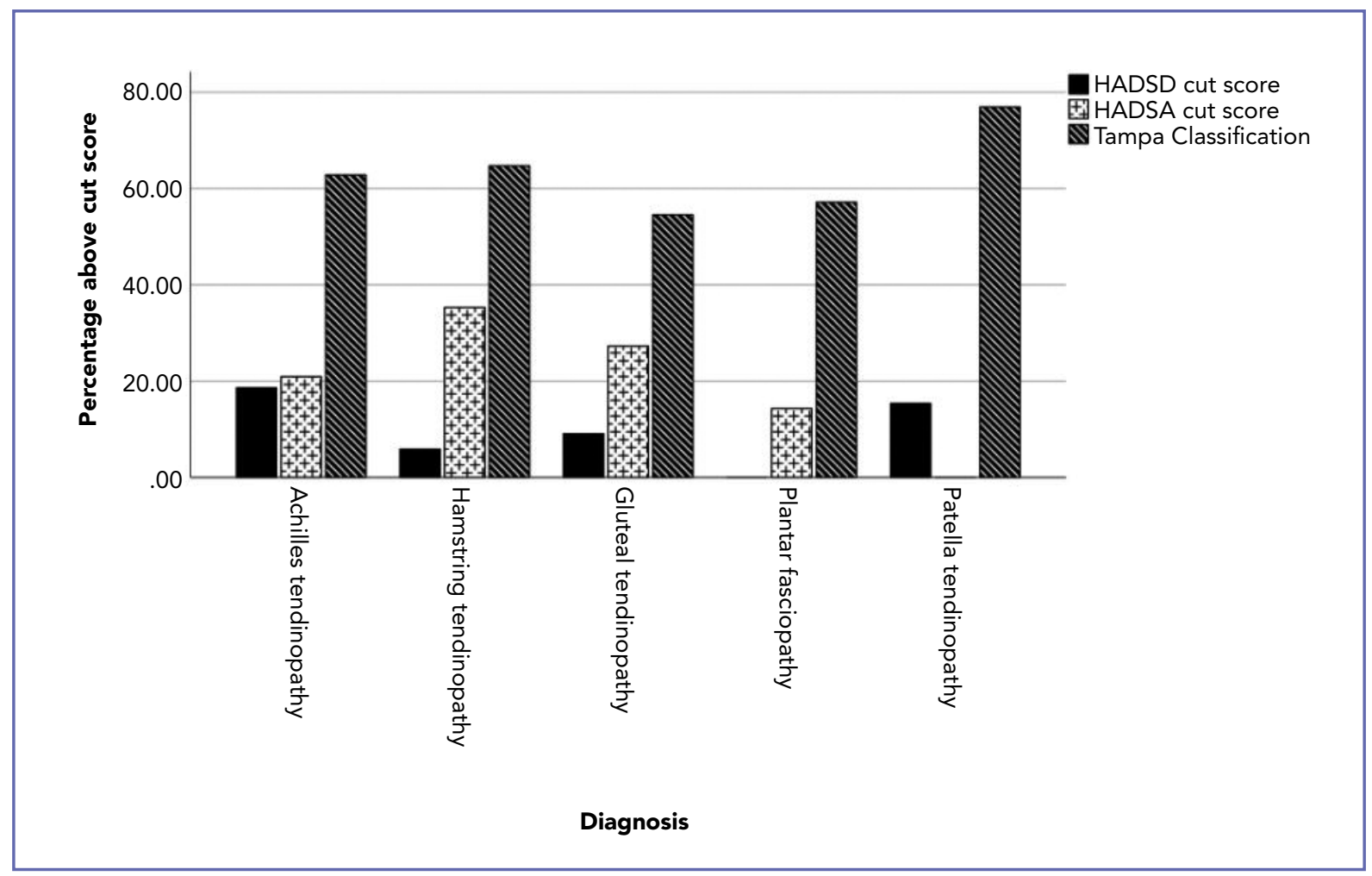

Figure 5. Participants classified with kinesiophobia, depression and anxiety.

prevalence ranging from $54.5 \%$ for gluteal tendinopathy to $76.9 \%$ for patellar tendinopathy.

Results of the current study suggest that those patients who displayed fear of movement according to the TSK, are more likely to hold the belief that pain is proportionate to damage, and that avoidance of physical activity or exercise may be necessary to prevent exacerbation of their tendinopathy condition (41). These patient cognitions are at odds with the current understanding of tendinopathy pathogenesis and management. Although the pain source in tendinopathy is not clear (42), the accepted hypothesis is that pain is a product of peripheral sensitisation that may be caused by one or a combination of multiple biochemical changes that occur in the local tissues (43). Pain is not always reflective of the state of the tissues and there is no evidence that tendon pain is an indication of structural tendon damage (3). There is also a substantial body of evidence that demonstrates structured exercise improves tendon pain and function, and exercise is the first-line recommended treatment for upper and lower limb tendinopathy $(1,4,44,45)$. Future studies should explore potential associations between kinesiophobia and treatment outcomes in lower limb tendinopathy.
Given the cross-sectional design of our study, we are unable to draw conclusions on the mechanistic relationship between kinesiophobia and tendon pain - whether fear of movement precedes or is a product of tendon pain. Our results also suggest there is a small negative association between pain severity (both at rest and with activity) and kinesophobia in patients with lower limb tendinopathies. The presence of kinesiophobia might form part of a multifaceted risk factor profile for the development of a chronic lower limb tendinopathy given this small association. One explanation for this could be that there is an underlying tendency towards kinesiophobia in some people and not in others, in a similar way that some people have a genetic or situational predisposition towards psychological conditions such as anxiety or depression (46). There is a body of evidence which demonstrates that fear-avoidance beliefs play a significant role in the transition from acute to chronic back pain (47) and is correlated to disability and quality of life measures (48). With respect to low back pain, high levels of kinesiophobia at baseline predicted duration, severity and disability at follow up. Further, for those without low back pain at baseline, a high level of kinesiophobia could predict back 
pain and disability at follow up (49). These results suggest a propensity to develop fear with movement could exist within a genotype of an individual, and that pain as an environmental trigger could lead to its phenotypic expression. This assertion is possibly supported by the negligible association between kinesiophobia and complaint duration in the current study suggesting other factors may be contributing the development of fear of movement. How these results translate to lower limb tendinopathies requires further exploration, however.

Alternatively, kinesiophobia could be thought of as a secondary sequalae to longstanding pain in some individuals. This could be explained as a maladaptive movement behavioural response to threatening pain, whereby the central nervous system interprets repeated nociceptive input from peripheral tissues, upregulates output pain and alters motor patterns to de-load the injured tissues (50-52). Whilst the temporal relationship between pain and fear of movement is unclear, our study suggests that identifying and managing these cognitions may have a role in the management of lower limb tendinopathies.

\section{Depression and anxiety}

Among our cohort, $13.2 \%$ ( $\mathrm{n}=12$ ) would be classified as experiencing depression and $20.9 \%(\mathrm{n}=19)$ experiencing anxiety, with the prevalence of both in the current cohort being relatively consistent with Australian population data (53). Pain at rest demonstrated a small association with the HADS depression subscale score, with depression being most prevalent in Achilles tendinopathy. The latter findings are consistent with the psychological burden among people with Achilles tendinopathy, as identified by McAuliffe et al. (54). Depression has also been identified as a significant component of the patient profile of those with severe gluteal tendinopathy (10), suggesting that clinicians should screen for psychological distress, or the presence of possible depression in patients with lower limb tendinopathies, to better manage these complaints (54). Anxiety appeared to be most prevalent in those with hamstring tendinopathy in the current study, but was not identified in those with patellar tendinopathy. The reason for these associations is not clear and further exploration in larger samples is warranted, particularly as these psychopathologies do not appear to be present to the same degree across all lower limb tendinopathies. As discussed earlier, whether psychological distress precedes, develops in conjunction with, or is exacerbated by, a lower limb tendinopathy requires further research.

\section{Life orientation}

The current study is the first to explore the construct of life orientation (dispositional optimism) in the context of lower limb tendinopathies. Optimism and pessimism comprise this construct with higher levels of pessimism being associated with lower physical health outcomes (55). Pessimism demonstrated a trivial negative correlation relationship and optimism a trivial positive relationship with duration of symptoms. Likewise, pain with either rest or activity demonstrated trivial correlations with dispositional optimism. These results suggest that life orientation may not be a factor in lower limb tendinopathies broadly. However, there may be a relationship between life orientation and individual tendinopathies that was not able to be identified given the sample size in the current study. Further work to evaluate the association of life orientation and specific lower limb tendinopathies to confirm or refute the current findings is required.

\section{Limitations}

There are several limitations of this study, the first being inclusion of people with concomitant musculoskeletal pathology such as hip osteoarthritis, patellofemoral pain syndrome or lower back pain as this may have influenced our findings. The presence of a secondary diagnoses may have resulted in over reporting of prevalence of psychological factors such as kinesiophobia. Another limitation of our study is that we did not use validated measures of tendinopathy pain and function (37-40). This is because there is no one measure that incorporates all lower limb tendinopathy diagnoses that we included in the study. This may have limited our ability to identify a relationship between severity of pain and psychological factors, which consequently may be under reported. In addition, our consecutive cohort was a chronic cohort presenting to a tertiary referral specialist clinic and may not represent patients with shorter term pain, presenting to primary and secondary care centres, or patients with these conditions who do not actively seek specialist treatment. Generalising our findings to other populations such as those who have never sought treatment for their tendinopathy, or those with acute symptom durations is not recommended.

We urge caution generalizing our findings, given the relatively small subgroups of some of the tendinopathies represented in our cohort. Given that ours is a pragmatic exploratory study we did not consider power calculations a-priori. Finally, there were very few cases of some tendinopathy diagnoses included in the study. Plantar fasciopathy was 
the lowest $(n=7)$. For this reason, we did not analyse relationships between tendinopathy types and associated pain measures. Consequently, there is a limit on generalisability of our results to individual tendinopathy types included in the study. Despite these limitations, our results demonstrate the high prevalence of kinesiophobia across all tendinopathy diagnoses among our consecutive cohort of patients. The authors therefore advocate that this psychological factor warrants further investigation in future research in the field of lower limb tendinopathy.

\section{CONCLUSIONS}

The current work explored a range of demographic, health behaviour, psychological and psychosocial variables in a cohort of patients presenting to physiotherapy for treatment of a lower limb tendinopathy. Regardless of the tendinopathy location, kinesophobia appeared to be prevalent in the majority of participants in the study. This finding would suggest that routinely screening for kinesophobia may be indicated, as it may provide valuable clinical information to incorporate into patient management strategies. This result also provides an opportunity to evaluate the impact of kinesophobia on treatment outcomes. Some of the current patient cohort also demonstrated possible depression and anxiety - again,

\section{REFERENCES}

1. Malliaras P, Cook J, Purdam C, Rio E. Patellar tendinopathy: clinical diagnosis, load management, and advice for challenging case presentations. J Orthop Sports Phys Ther 2015:45(11):887-98.

2. Cook JL, Purdam CR. Is tendon pathology a continuum? A pathology model to explain the clinical presentation of load-induced tendinopathy. Br J Sports Med 2009;43(6):409-16.

3. Cook JL, Rio E, Purdam CR, Docking SI. Revisiting the continuum model of tendon pathology: what is its merit in clinical practice and research? Br J Sports Med 2016;50(19):1187-91.

4. Cardoso TB, Pizzari T, Kinsella R, Hope D, Cook JL. Current trends in tendinopathy management. Best Pract Res Clin Rheumatol 2019;33(1):122-40.

5. Thorpe CT, Riley GP, Birch HL, Clegg PD, Screen HRC. Fascicles and the interfascicular matrix show decreased fatigue life with ageing in energy storing tendons. Acta Biomater 2017;56:58-64.

6. Di Filippo L, Pennella D, Maselli F, Arrigoni P. Research proposal of a new clinic model for the interpretation of Lateral Elbow Pain: is it time to change? MLTJ 2020;10(1).

7. Vlaeyen JWS, Linton SJ. Fear-avoidance and its consequences in chronic musculoskeletal pain: a state of the art. Pain 2000;85(3):317-32.

8. Crofford LJ. Psychological aspects of chronic musculoskeletal pain. Best Pract Res Clin Rheumatol 2015;29(1):147-55.

9. Drake C, Mallows A, Littlewood C. Psychosocial variables and presence, severity and prognosis of plantar heel pain: A screening and co-management of these psychopathologies may be required to effectively manage patients with lower limb tendinopathy. Of note is that these psychopathologies appeared to be independent of other demographic variables in this cohort. The current study adds to the understanding of the prevalence and associations between lower limb tendinopathies and a range of psychological and psychosocial variables. These results could provide the basis for further work to evaluate the impact of addressing psychological and psychosocial variables in the management of lower limb tendinopathies. Further research could be directed towards tracking psychological changes during the management of a tendinopathy through to resolution, including the use of other measures that observe coping, self-management behaviours and self-efficacy. These additional measures may assist in the identification of factors that predict chronicity and poor treatment outcomes. Whilst quality of life and functional measures were not included in this study, they should be included in future studies to enable a more complete exploration of the relationship between tendinopathy and psychological factors.

\section{CONFLICT OF INTERESTS}

The authors declare that they have no conflict of interests. systematic review of cross-sectional and prognostic associations. Musculoskelet Care 2018;16(3):329-38.

10. Plinsinga ML, Coombes BK, Mellor R, et al. Psychological factors not strength deficits are associated with severity of gluteal tendinopathy: A cross-sectional study. European Journal of Pain 2018;22(6):1124-33.

11. Silbernagel KG, Brorsson A, Lundberg M. The majority of patients with Achilles tendinopathy recover fully when treated with exercise alone: a 5-year follow-up. Am J Sports Med 2011;39(3):607-13.

12. Jayaseelan DJ, Weber MJ, Jonely H. Potential Nervous System Sensitization in Patients With Persistent Lower Extremity Tendinopathies: 3 Case Reports. J Orthop Sports Phys Ther 2019.

13. Padulo J, Oliva F, Frizziero A, Maffulli N. Muscle, Ligaments and Tendons Journal - Basic principles and recommendations in clinical and field science research: 2016 update. MLTJ 2016;6(1):1-5.

14. Vaughan B, Fitzgerald K, Fleischmann M, Mulcahy J. Determinants of health, health behaviours and demographic profile of patients attending an Australian university student-led osteopathy clinic. Chiropr Man Therap 2020;28(1):2.

15. Zigmond AS, Snaith RP. The hospital anxiety and depression scale. Acta Psychiatr Scand 1983;67(6):361-70.

16. Smarr KL, Keefer AL. Measures of depression and depressive symptoms: beck depression inventory-II (BDI-II), Center for Epidemiologic Studies Depression Scale (CES-D), geriatric depression scale (GDS), hospital anxiety and depression scale 
(HADS), and patient health Questionnaire-9 (PHQ-9). Arthritis Care Res (Hoboken) 2011;63(S11):S454-S66.

17. Bjelland I, Dahl AA, Haug TT, Neckelmann D. The validity of the Hospital Anxiety and Depression Scale: an updated literature review. J Psychosom Res 2002;52(2):69-77.

18. Cameron IM, Crawford JR, Lawton K, Reid IC. Psychometric comparison of PHQ-9 and HADS for measuring depression severity in primary care. Br J Gen Pract 2008;58(546):32-6.

19. Hansson M, Chotai J, Nordstöm A, Bodlund O. Comparison of two self-rating scales to detect depression: HADS and PHQ-9. Br J Gen Pract 2009;59(566):e283-e8.

20. Johnston M, Wright S, Weinman J. Measures in health psychology : a user's portfolio. Windsor: NFER-NELSON, 1995.

21. Hudes K. The Tampa Scale of Kinesiophobia and neck pain, disability and range of motion: a narrative review of the literature. J Can Chiropr Assoc 2011;55(3):222.

22. Vlaeyen JWS, Kole-Snijders AMJ, Boeren RGB, Van Eek H. Fear of movement/(re) injury in chronic low back pain and its relation to behavioral performance. Pain 1995;62(3):363-72.

23. Lundberg MKE, Styf J, Carlsson SG. A psychometric evaluation of the Tampa Scale for Kinesiophobia-from a physiotherapeutic perspective. Physiother Theory Pract 2004;20(2):121-33.

24. Bunketorp L, Carlsson J, Kowalski J, Stener-Victorin E. Evaluating the reliability of multi-item scales: A non-parametric approach to the ordered categorical structure of data collected with the Swedish version of the Tampa Scale for Kinesiophobia and the Self-efficacy Scale. J Rehabil Med 2005;37(5): 330-4.

25. French DJ, France CR, Vigneau F, French JA, Evans RT. Fear of movement/(re) injury in chronic pain: a psychometric assessment of the original English version of the Tampa scale for kinesiophobia (TSK). Pain 2007;127(1-2):42-51.

26. Herzberg PY, Glaesmer H, Hoyer J. Separating optimism and pessimism: A robust psychometric analysis of the Revised Life Orientation Test (LOT-R). Psychol Assess 2006;18(4):433-8.

27. Scheier MF, Carver CS. Optimism, coping, and health: assessment and implications of generalized outcome expectancies. Health Psychol 1985;4(3):219-47.

28. Glaesmer H, Rief W, Martin A, et al. Psychometric properties and population-based norms of the Life Orientation Test Revised (LOT-R). Br J Health Psychol 2012;17(2):432-45.

29. Miller RP, Kori SH, Todd DD. The Tampa Scale. Unpublished report. Tampa, Florida, 1991.

30. Riel H, Vicenzino B, Jensen MB, Olesen JL, Holden S, Rathleff MS. The effect of isometric exercise on pain in individuals with plantar fasciopathy: A randomized crossover trial. Scand J Med Sci Sports 2018;28(12):2643-50.

31. Goom TSH, Malliaras P, Reiman MP, Purdam CR. Proximal hamstring tendinopathy: clinical aspects of assessment and management. J Orthop Sports Phys Ther 2016;46(6):483-93.

32. Grimaldi A, Mellor R, Hodges P, Bennell K, Wajswelner H, Vicenzino B. Gluteal tendinopathy: a review of mechanisms, assessment and management. Sports Med 2015;45(8):1107-19.

33. Grimaldi A, Fearon A. Gluteal tendinopathy: integrating pathomechanics and clinical features in its management. J Orthop Sports Phys Ther 2015;45(11):910-22.

34. Reiman M, Burgi C, Strube E, et al. The utility of clinical measures for the diagnosis of achilles tendon injuries: a systematic review with meta-analysis. J Athl Train 2014;49(6):820-9.
35. Menz HB, Thomas MJ, Marshall M, et al. Coexistence of plantar calcaneal spurs and plantar fascial thickening in individuals with plantar heel pain. Rheumatology 2018;58(2):237-45.

36. Scott A, Backman LJ, Speed C. Tendinopathy: update on pathophysiology. J Orthop Sports Phys Ther 2015;45(11):833-41.

37. Visentini PJ, Khan KM, Cook JL, et al. The VISA score: an index of severity of symptoms in patients with jumper's knee (patellar tendinosis). J Sci Med Sport 1998;1(1):22-8.

38. Cacchio A, De Paulis F, Maffulli N. Development and validation of a new visa questionnaire (VISA-H) for patients with proximal hamstring tendinopathy. Br J Sports Med 2014;48(6):448-52.

39. Fearon AM, Ganderton C, Scarvell JM, et al. Development and validation of a VISA tendinopathy questionnaire for greater trochanteric pain syndrome, the VISA-G. Man Ther 2015;20(6):805-13.

40. Robinson JM, Cook JL, Purdam C, et al. The VISA-A questionnaire: a valid and reliable index of the clinical severity of Achilles tendinopathy. Br J Sports Med 2001;35(5):335-41.

41. Fearon A, Neeman T, Smith P, Scarvell J, Cook J. Pain, not structural impairments may explain activity limitations in people with gluteal tendinopathy or hip osteoarthritis: a cross sectional study. Gait Posture 2017;52:237-43.

42. Rio E, Moseley L, Purdam C, et al. The pain of tendinopathy: physiological or pathophysiological? Sports Med 2014;44(1):9-23.

43. Plinsinga ML, van Wilgen CP, Brink MS, et al. Patellar and Achilles tendinopathies are predominantly peripheral pain states: a blinded case control study of somatosensory and psychological profiles. Br J Sports Med 2018;52(5):284-91.

44. Malliaras P, Barton CJ, Reeves ND, Langberg H. Achilles and Patellar Tendinopathy Loading Programmes. Sports Med 2013;43(4):267-86.

45. Färnqvist K, Malliaras P, Pearson S. Eccentric Exercise, Tendon Thickness, Pain and Function in Achilles Tendinopathy: A Systematic Review. J Sport Rehabil 2019:1-30.

46. Burri A, Ogata S, Livshits G, Williams F. The association between chronic widespread musculoskeletal pain, depression and fatigue is genetically mediated. PLoS One 2015;10(11):e0140289.

47. Buer N, Linton SJ. Fear-avoidance beliefs and catastrophizing: occurrence and risk factor in back pain and ADL in the general population. Pain 2002;99(3):485-91.

48. Comachio J, Magalhães MO, Carvalho APdMC, Marques AP. A cross-sectional study of associations between kinesiophobia, pain, disability, and quality of life in patients with chronic low back pain. Adv Rheumatology 2018;58(1):8.

49. Picavet HSJ, Vlaeyen JWS, Schouten JSAG. Pain catastrophizing and kinesiophobia: predictors of chronic low back pain. Am J Epidemiol 2002;156(11):1028-34.

50. O'Sullivan P. Diagnosis and classification of chronic low back pain disorders: maladaptive movement and motor control impairments as underlying mechanism. Man Ther 2005;10(4):242-55.

51. Linton SJ. A review of psychological risk factors in back and neck pain. Spine (Phila Pa 1976) 2000;25(9):1148-56.

52. Zusman M. Forebrain-mediated sensitization of central pain pathways:'non-specific'pain and a new image for MT. Man Ther 2002;7(2):80-8. 
53. Australian Bureau of Statistics. National Survey of Mental Health and Wellbeing. Canberra: Australian Bureau of Statistics, 2008.

54. Mc Auliffe S, Synott A, Casey H, Mc Creesh K, Purtill H, O'Sullivan K. Beyond the tendon: experiences and perceptions of people with persistent Achilles tendinopathy. Musculoskeletal Sci Pract 2017;29:108-14.

55. Rasmussen HN, Scheier MF, Greenhouse JB. Optimism and physical health: A meta-analytic review. Ann Behav Med 2009;37(3):239-56. 


\section{SUPPLEMENTARY FILE 1}

Table I Suppl. Clinical diagnosis criteria for tendinopathy.

\begin{tabular}{ll}
\hline Site of tendinopathy & Clinical diagnosis criteria \\
\hline Gluteal & $\begin{array}{l}\text { Primary complaint of lateral hip pain, pain on palpation of the greater trochanter, and pain reproduced } \\
\text { with either passive flexion abduction external rotation (FADER), muscle isometric contraction in } \\
\text { FADER or single leg stance on the affected leg for 30 seconds }(1,2)\end{array}$ \\
\hline Proximal hamstring & $\begin{array}{l}\text { Primary complaint of ischial tuberosity pain, pain on single leg bridge, single leg long lever bridge or } \\
\text { single leg deadlift loading tests (3) }\end{array}$ \\
\hline Patella & $\begin{array}{l}\text { Primary complaint of localised pain at the inferior pole of the patellar, corresponding tenderness on } \\
\text { palpation, pain on single leg decline squat or submaximal hop loading tests (4) }\end{array}$ \\
\hline Achilles & $\begin{array}{l}\text { Primary complaint of Achilles insertion or midportion pain, corresponding tenderness on palpation, } \\
\text { pain on calf raise or submaximal hop loading tests (5) }\end{array}$ \\
\hline Plantar fascia & $\begin{array}{l}\text { Primary complaint of pain at the proximal plantar fascia insertion, corresponding pain on palpation, } \\
\text { pain on calf raise or submaximal hop loading tests (6) }\end{array}$ \\
\hline
\end{tabular}

\section{REFERENCES}

1. Grimaldi A, Fearon A. Gluteal Tendinopathy: Integrating Pathomechanics and Clinical Features in Its Management. J Orthop Sports Phys Ther 2015;45(11):910-22.

2. Grimaldi A, Mellor R, Hodges P, Bennell K, Wajswelner H, Vicenzino B. Gluteal Tendinopathy: A Review of Mechanisms, Assessment and Management. Sports Med 2015;45(8):1107-19.

3. Goom TS, Malliaras P, Reiman MP, Purdam CR. Proximal Hamstring Tendinopathy: Clinical Aspects of Assessment and Management. J Orthop Sports Phys Ther 2016;46(6):483-93.

4. Malliaras P, Cook J, Purdam C, Rio E. Patellar Tendinopathy: Clinical Diagnosis, Load Management, and Advice for
Challenging Case Presentations. J Orthop Sports Phys Ther 2015;45(11):887-98.

5. Reiman M, Burgi C, Strube E, Prue K, Ray K, Elliott A, Goode A. The utility of clinical measures for the diagnosis of achilles tendon injuries: a systematic review with meta-analysis. J Athl Train 2014;49(6):820-9.

6. Menz HB, Thomas MJ, Marshall M, et al. Coexistence of plantar calcaneal spurs and plantar fascial thickening in individuals with plantar heel pain. Rheumatology (Oxford) 2019;58(2):237-245. 


\section{SUPPLEMENTARY FILE 2}

Table II Suppl. Differential diagnoses considered (*systemic \& sinister pathologies considered and screened for in all cases).

\begin{tabular}{|c|c|}
\hline Site of tendinopathy & Differential diagnosis \\
\hline Gluteal & $\begin{array}{l}\text { Lumbar stenosis, radiculopathy or discogenic pathology }(1,2) \\
\text { Sacroiliac joint dysfunction (3) } \\
\text { Hip joint pathology and osteoarthritis }(4) \\
\text { Ischiofemoral impingement }(4) \\
\text { Neck of femur fracture }(2) \\
\text { Sciatic neuropathy }(2)\end{array}$ \\
\hline Patellar & $\begin{array}{l}\text { Patellofemoral joint dysfunction (8) and osteoarthritis (9) } \\
\text { Patellar inferior pole bone stress injury and osteochondroses of the knee (10) } \\
\text { Infrapatellar fat pad (11) } \\
\text { Plica and chondral surface pathology }(12,13)\end{array}$ \\
\hline Achilles & $\begin{array}{l}\text { Sural neuropathy }(14) \\
\text { Paratenon (15) } \\
\text { Tendon partial tear, rupture (16) } \\
\text { Plantaris tendinopathy }(17)\end{array}$ \\
\hline Plantar fascia & $\begin{array}{l}\text { Tibial neuropathy }(18,19) \\
\text { Calcaneum bone stress injury }(18,19) \\
\text { Fat pad contusion }(18,19)\end{array}$ \\
\hline
\end{tabular}

\section{REFERENCES}

1. Cook C, Hegedus E. Diagnostic utility of clinical tests for spinal dysfunction. Man Ther 2011;16(1):21-5.

2. Grimaldi A, Fearon A. Gluteal Tendinopathy: Integrating Pathomechanics and Clinical Features in Its Management. J Orthop Sports Phys Ther 2015;45(11):910-22.

3. Laslett M, Aprill CN, McDonald B, Young SB. Diagnosis of sacroiliac joint pain: validity of individual provocation tests and composites of tests. Man Ther 2005;10(3):207-18.

4. Grimaldi A, Mellor R, Hodges P, Bennell K, Wajswelner H, Vicenzino B. Gluteal Tendinopathy: A Review of Mechanisms, Assessment and Management. Sports Med 2015;45(8):1107-19.

5. Goom TS, Malliaras P, Reiman MP, Purdam CR. Proximal Hamstring Tendinopathy: Clinical Aspects of Assessment and Management. J Orthop Sports Phys Ther 2016;46(6):483-93.

6. Laslett M. Clinical Diagnosis of Sacroiliac Joint Pain. Tech Orthop 2019;34(2):76-86.

7. Punnoose A R, Lynm C, Golub RM. Slipped Capital Femoral Epiphysis. JAMA 2013;309(6):620-620.

8. Post WR, Fulkerson J. Knee pain diagrams: correlation with physical examination findings in patients with anterior knee pain. Arthroscopy 1994;10(6):618-23.

9. Crossley KM. Is patellofemoral osteoarthritis a common sequela of patellofemoral pain? BMJ Publishing Group Ltd and British Association of Sport and Exercise Medicine, 2014.

10. Gholve PA, Scher DM, Khakharia S, Widmann RF, Green DW. Osgood Schlatter syndrome. Curr Opin Pediatr 2007;19(1):44-50.
11. Dragoo JL, Johnson C, McConnell J. Evaluation and treatment of disorders of the infrapatellar fat pad. Sports Med 2012;42(1):51-67.

12. Schindler OS. 'The Sneaky Plica' revisited: morphology, pathophysiology and treatment of synovial plicae of the knee. Knee Surg Sports Traumatol Arthrosc 2014;22(2):247-62.

13. Malliaras P, Cook J, Purdam C, Rio E. Patellar Tendinopathy: Clinical Diagnosis, Load Management, and Advice for Challenging Case Presentations. J Orthop Sports Phys Ther 2015;45(11):887-98.

14. Coppieters MW, Crooke JL, Lawrenson PR, Khoo SJ, Skulstad T, Bet-Or Y. A modified straight leg raise test to differentiate between sural nerve pathology and Achilles tendinopathy. A cross-sectional cadaver study. Man Ther 2015;20(4):587-91.

15. Reiman M, Burgi C, Strube E, Prue K, Ray K, Elliott A, Goode A. The utility of clinical measures for the diagnosis of achilles tendon injuries: a systematic review with meta-analysis. J Athl Train 2014;49(6):820-9.

16. Maffulli N. The clinical diagnosis of subcutaneous tear of the Achilles tendon. Am J Sports Med 1998;26(2):266-270.

17. Alfredson H. Persistent pain in the Achilles mid-portion? Consider the plantaris tendon as a possible culprit! Br J Sports Med 2017;51(10):833-834.

18. Rompe JD. Plantar fasciopathy. Sports Med Arthrosc Rev 2009;17(2):100-4.

19. Neufeld SK, Cerrato R. Plantar fasciitis: evaluation and treatment. J Am Acad Orthop Surg 2008;16(6):338-46. 